INPLASY

PROTOCOL

To cite: Zen et al. Survival Outcomes and Safety of PD-1/ PD-L1 Inhibitors for Unresectable Hepatocellular Carcinoma: Result from Phase III Trials. Inplasy protocol 202210067. doi: 10.37766/inplasy2022.1.0067

Received: 13 January 2022

Published: 14 January 2022

Corresponding author: Wei Yu

wyu@zju.edu.cn

Author Affiliation:

The First Affiliated Hospital, Zhejiang University School of Medicine.

Support: No.

Review Stage at time of this submission: Data analysis Completed but not published.

Conflicts of interest:

None declared.

\section{Survival Outcomes and Safety of PD-1/ PD-L1 Inhibitors for Unresectable Hepatocellular Carcinoma: Result from Phase III Trials}

\author{
Zen, LY1; Su, JW2; Qiu, WQ3; Jin, XH4; Qiu, YQ5; Yu, W6.
}

Review question / Objective: The aim of this study was conducted a meta-analysis to assess the efficacy and safety of programmed cell death (PD-1) and programmed cell death ligand 1 (PD-L1) inhibitors in patients with unresectable hepatocellular carcinoma (UHCC).

Condition being studied: PD-1/PD-L1 inhibitors in multiple phase II/III trials have been demonstrated to improve the prognosis of patients with advanced cancers, such as melanoma, renal cell carcinoma, non-small cell lung cancer, urothelial bladder cancer. The efficacy of PD-1/PD-L1 inhibitors monotherapy or combination is variable for uHCC in phase II trials. However, for patients with uHCC, the improvements of OS in phase II and III trials were not complete coincident. In addition, only a minority of patients have durable response. Furthermore, toxicities including immune-related adverse events (irAEs) are increasingly being reported, posing therapeutic challenges for clinicians.

INPLASY registration number: This protocol was registered with the International Platform of Registered Systematic Review and Meta-Analysis Protocols (INPLASY) on 14 January 2022 and was last updated on 14 January 2022 (registration number INPLASY202210067).

\section{INTRODUCTION}

Review question / Objective: The aim of this study was conducted a meta-analysis to assess the efficacy and safety of programmed cell death (PD-1) and programmed cell death ligand 1 (PD-L1) inhibitors in patients with unresectable hepatocellular carcinoma (uHCC).
Condition being studied: PD-1/PD-L1 inhibitors in multiple phase II/III trials have been demonstrated to improve the prognosis of patients with advanced cancers, such as melanoma, renal cell carcinoma, non-small cell lung cancer, urothelial bladder cancer. The efficacy of PD-1/PD-L1 inhibitors monotherapy or combination is variable for uHCC in phase 
II trials. However, for patients with uHCC, the improvements of OS in phase II and III trials were not complete coincident. In addition, only a minority of patients have durable response. Furthermore, toxicities including immune-related adverse events (irAEs) are increasingly being reported, posing therapeutic challenges for clinicians.

\section{METHODS}

Participant or population: Unresectable hepatocellular carcinoma.

Intervention: PD-1/PD-L1 inhibitors monotherapy or combination.

Comparator: Sorafenib and placebo

Study designs to be included: Phase III clinical trials.

Eligibility criteria: Literatures met the following predetermined criteria were eligible for inclusion: (1) the patients with uHCC received PD-1/PD-L1 inhibitors; (2) phase III randomized controlled studies (RCTs); (3) sufficient data of efficacy outcomes including OS, progression-free survival (PFS), objective response rate (ORR), disease control rate (DCR); (4) data available on rates of adverse effects (AEs) from any cause, grade 3-4 AEs, or serious adverse events (SAEs).American Society of Clinical Oncology.

Information sources: Six databases of PubMed, Embase, Cochrane Library, Web of Science, Scopus and ClinicalTrials.gov.

Main outcome(s): Efficacy outcomes including overall survival (OS), progressionfree survival (PFS), objective response rate (ORR), disease control rate (DCR).

Quality assessment / Risk of bias analysis: Risk of bias in the included studies was evaluated with Jadad score.

Strategy of data synthesis: The data of hazard ratio (HR) with $95 \%$ confidence intervals (CI) from each study was extracted. After logarithmic transformation,
effect/CI was used to calculate pooled effect of HR by random effect models. The heterogeneity was evaluated by I-squared statistic. I-squared value greater than $50 \%$ suggested high heterogeneity. All of these were performed by STATA version 14 (STATA Corp, College Station, TX, USA).

Subgroup analysis: Subgroup analysis of OS and PFS were performed according to sex, geographic region, Barcelona Clinic Liver Cancer (BCLC) status and viral status.Subgroup analyses were further explored according to varied factors.

Sensitivity analysis: Sensitivity analysis was used to estimate the influence of a single study on the overall results of the metaanalysis.

Country(ies) involved: China.

Keywords: PD-1, PD-L1, hepatocellular carcinoma, survival, adverse effects.

Contributions of each author:

Author 1 - Linyan Zeng.

Author 2 - Junwei Su.

Author 3 - Wenqi Qiu.

Author 4 - Xuehang Jin.

Author 5 - Yunqing Qiu.

Author 6 - Wei Yu. 